\title{
Progressive imaging: S-transform order
}

\author{
H. Zhu* R. A. Brown ${ }^{\dagger} \quad$ R. J. Villanueva \\ J. Villanueva-Oller ${ }^{\ddagger}$ M. L. Lauzon* J. R. Mitchell* \\ A. G. Law*
}

(Received 8 August 2003; revised 17 December 2003)

\begin{abstract}
The paper focuses on progressive transmission of CT or MR images, and introduces two general schemes that are built around information embedded in transforms of images. A direct, a priori ordering of 93, parallel, CT slices of a head is obtained by successively finer sweepings of their natural subscript ordering to give a benchmark illustration. By comparison, an ordering of these CT slices simply by their energies is seen to not provide a viable progressive imaging scheme, at least when an overall, 3D skin level rendering is the gauge employed.
\end{abstract}

*Departments of Radiology and Clinical Neurosciences, University of Calgary, CANADA. mailto:hzhu@ucalgary.ca

${ }^{\dagger}$ Department of Electrical and Computer Engineering, University of Calgary, CANADA

${ }_{\ddagger}^{\ddagger}$ Instituto de Matemática Multidisciplinar, Universidad Poletécnica de Valencia, SPAIN

See http://anziamj.austms.org.au/V45/CTAC2003/Zhu1/home.html for this article, (c) Austral. Mathematical Soc. 2004. Published September 7, 2004. ISSN 1446-8735 
To investigate progressive imaging that does not obscure internal detail, two techniques based on transform space information are introduced here, and illustrated in detail with a $128 \times 128 \mathrm{MR}$ slice of a head $I(x, y)$. The first uses decreasing size of the moduli of the elements of the Fourier transform $F\left(k_{x}, k_{y}\right)$ of $I(x, y)$. The second, a one parameter generalization, exploits the localization feature of the recent $S$-transform and also provides a capability for an observer to outline a region of interest within the progressive transmission process. Both transform based methods are effective for the specific illustrations included, and the latter opens important research questions for application in analysis, handling or interpretation of the massive data sets arising in magnetic resonance imaging.

\section{Contents}

1 Introduction

C1003

2 The $S$-transform: Structure, features and computation C1005

3 Progressive imaging: Transform space ordering C1006

4 Concluding comments

C1015

References

C1016

\section{Introduction}

In medical as well as other applications areas of imaging science, differing approaches for handling large, 3D digital objects are under investigation. As technology evolves, the data sets captured for subsequent analysis, interpretation, rendering or transmission burgeon in size. For example, cur- 
rent spiral-CT scanners can image hundreds of slices per exam and produce 100 Mbytes or more of data, while magnetic resonance (MR) or 3D ultrasound imaging are capable of capturing 500 Mbytes of data, in just a few minutes.

For large amounts of 3D data, their network transmission is approached in different ways. One class of techniques, and this is the scenario for the development below, hinges on progressive transmission of constituent subsets of the object, for subsequent emerging renderings. During progressive viewing at the recipient node,

1. if the evolving renderings indicate that the object seems to be desirable, then only the remainder of the data need be transmitted, whereas

2. if it is not appearing to be needed, transmission can be terminated.

As well, if some subfeature is emerging as important, the recipient can interrupt the process and initiate transmission of contiguous subdata instead. Recent mathematical machinery for progressive transmission of a digital object includes decorrelation techniques [3], wavelets and matrix polynomials [2]. The scheme introduced here is based, instead, on producing an ordering of constituent subsets of the object that is developed from analysis of a transform of the object. The Fourier transform (FT) is used first, then development is extended to exploit the recently introduced $S$-transform (ST) which can be effective for uncovering localized detail [5].

Section 2 provides a summary of the ST and its inverse, including its computation. Ordering for progressive imaging is the main focus of Section 3: a direct, binary sweeping scheme for a "parallel slices" decomposition of a human head is displayed. Then, by contrast, "energy" orderings using a transform are developed and illustrated. Section 4 includes some final comments and ideas for future research. 


\section{The $S$-transform: Structure, features and computation}

The Fourier transform is a powerful tool with wide usage within a variety of areas of analysis or application. In medical informatics, it not only plays vital roles for post-processing medical data but, in light of the Fourier Slice Theorem, it also forms the cornerstone of different imaging modalities, including magnetic resonance imaging and computed tomography (CT). In the $2 \mathrm{D}$ setting, the FT, $\mathcal{F}$, of an image $I(x, y)$ is the function

$$
F\left(k_{x}, k_{y}\right)=\mathcal{F}\{I\}\left(k_{x}, k_{y}\right)=\int_{-\infty}^{\infty} \int_{-\infty}^{\infty} I(x, y) \exp \left[-2 \pi i\left(k_{x} x+k_{y} y\right)\right] d x d y .
$$

A discretized version of (1) is invariably computed through a loop which exploits a fast Fourier transform (FFT) evaluation of the 1D FT.

An FT decomposes a signal (1D), image (2D), or volume (3D) into sinusoids of different frequencies, but does not, however, reveal when or where ${ }^{1}$ a particular frequency component may occur. Like the Gabor transform (GT) and wavelet transform (WT), the recent $S$-transform is a useful mathematical mechanism for extracting information about time varying or space varying frequencies $[5,6]$. The ST has a structure clearly related to that of the FT, and combines the time-frequency representation of the GT with the multiscale feature of the WT [5]. In addition, the Fourier and $S$ spectra can be readily converted from one to the other, hence the processing of medical or other data in ST space may lead to further tools for effective use, or transmission, of data.

${ }^{1}$ In MRI, for example, subject motion, respiratory/cardiac activities, blood flow and other physiological fluctuations can result in data which vary spatially or temporally. 
The 2D $S$-transform of an image $I(x, y)$ is the $4 \mathrm{D}$ function [5]

$$
\begin{gathered}
S\left(x, y, k_{x}, k_{y}\right)=\frac{\left|k_{x}\right|\left|k_{y}\right|}{2 \pi} \int_{-\infty}^{\infty} \int_{-\infty}^{\infty} I\left(x^{\prime}, y^{\prime}\right) \exp \left[-\frac{\left(x^{\prime}-x\right)^{2} k_{x}^{2}+\left(y^{\prime}-y\right)^{2} k_{y}^{2}}{2}\right] \\
\times \exp \left[-2 \pi i\left(k_{x} x^{\prime}+k_{y} y^{\prime}\right)\right] d x^{\prime} d y^{\prime} .
\end{gathered}
$$

A direct calculation confirms that the inverse of (2) is

$$
I(x, y)=\mathcal{F}^{-1}\left\{\int_{-\infty}^{\infty} \int_{-\infty}^{\infty} S\left(x, y, k_{x}, k_{y}\right) d x d y\right\} .
$$

Also, through use of the shift property and the Fourier Convolution Theorem, (2) can be expressed in the alternative form

$$
\begin{gathered}
S\left(x, y, k_{x}, k_{y}\right)=\int_{-\infty}^{\infty} \int_{-\infty}^{\infty} F\left(\alpha+k_{x}, \beta+k_{y}\right) \exp \left[-2 \pi^{2}\left(\frac{\alpha^{2}}{k_{x}^{2}}+\frac{\beta^{2}}{k_{y}^{2}}\right)\right] \\
\times \exp [2 \pi i(\alpha x+\beta y)] d \alpha d \beta,
\end{gathered}
$$

where $F\left(k_{x}, k_{y}\right)$ is the Fourier transform of $I(x, y)$. It is this form which serves as the basis for an algorithm that utilizes the FFT repeatedly for computation of the ST, as indicated in Algorithm 1 [1].

Progressive transmission of an image with orderings determined using its FT, or its ST, are developed and illustrated in the next section.

\section{Progressive imaging: Transform space ordering}

The general process of progressive transmission of images will first be illustrated using a simple "binary sweeping", a priori ordering scheme with a test data set consisting of 93 parallel CT slices of a human head [4], each slice of resolution $128 \times 128$. Denote this digital object as

$$
\left\{\left(x_{s}, N_{s}\right)\right\}_{s=0}^{92},
$$


Algorithm 1 Computation of the ST of $I(x, y)$ according to (4)

1: Fast Fourier transform the image: $I(x, y) \rightarrow F(\alpha, \beta)$

2: for all frequency $\left(k_{x}, k_{y}\right)$ (where $\left.k_{x}, k_{y} \neq 0\right)$ do

3: Compute the frequency domain Gaussian localizing window at the current frequency $\left(k_{x}, k_{y}\right): \exp \left[-2 \pi^{2}\left(\frac{\alpha^{2}}{k_{x}^{2}}+\frac{\beta^{2}}{k_{y}^{2}}\right)\right] \rightarrow W(\alpha, \beta)$

4: $\quad$ Shift the Fourier spectrum $F(\alpha, \beta)$ to $F\left(\alpha+k_{x}, \beta+k_{y}\right)$

5: $\quad$ Compute the point-wise multiplication of $F\left(\alpha+k_{x}, \beta+k_{y}\right)$ and $W(\alpha, \beta)$, and denote it as $M_{k_{x}, k_{y}}(\alpha, \beta)$

6: Inverse FFT $M_{k_{x}, k_{y}}(\alpha, \beta)$ to provide the spatial location of events corresponding to frequency, that is, $M_{k_{x}, k_{y}}(\alpha, \beta) \stackrel{I F F T}{\longrightarrow} S_{k_{x}, k_{y}}(x, y)$

7: end for

8: For the frequencies $\left(k_{x}, 0\right)$ and $\left(0, k_{y}\right)$, the Gaussian window function becomes $\exp \left(-2 \pi^{2} \alpha^{2} / k_{x}^{2}\right)$ and $\exp \left(-2 \pi^{2} \beta^{2} / k_{y}^{2}\right)$, respectively, then Steps 4-6 are computed.

9: For the frequency $(0,0), S_{0,0}(x, y)$ is simply the mean of $I(x, y)$.

where $x_{0}<x_{1}<x_{2}<\cdots<x_{92}$ provide ordering of the slices, from top to bottom of the head, and the $N_{s}$ are $128 \times 128$ matrices. One simple, direct strategy for determining a slice ordering involves repeated binary sweepings [2]: $N_{0}$ and $N_{92}$ are transmitted initially and, thereafter, the subscripts selected by the next top to bottom sweep are the ones determined as the midpoints between the slice locations used so far ${ }^{2}$. This selection process is independent of the internal data.

Figure 1 illustrates the binary sweepings scheme with the data set (5). The renderings were computed from a matrix cubic spline technique [2], each at the end of a full sweep. For example, after 29 of 93 slices (that is, $31.2 \%$ of the original data) were transmitted, the corresponding rendering prescribed at skin level appears in Figure 1b. The original 93-slice data set is displayed in Figure 1f. There is an important point to make with

${ }^{2}$ If there were 64 slices, the ordering would thus be 0,$63 ; 32 ; 16,48 ; 8,24,40,56 ; \ldots$ 


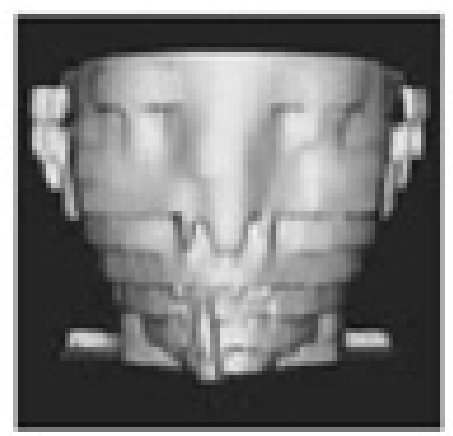

(a) $11.8 \%$

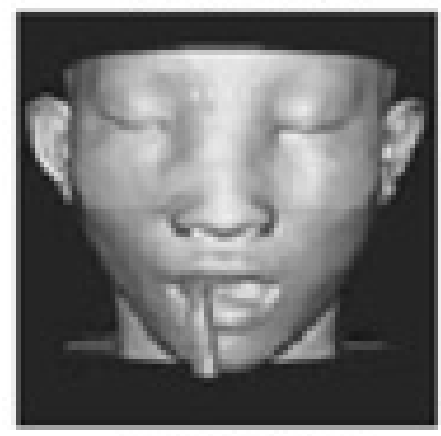

(d) $69.9 \%$

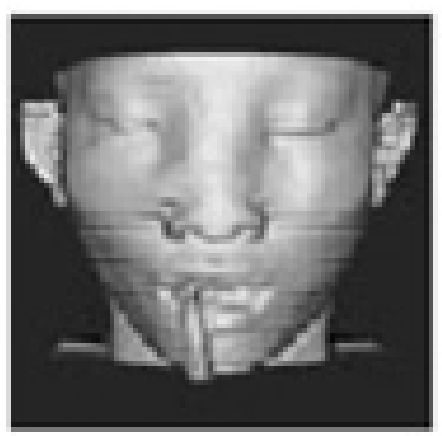

(b) $31.2 \%$

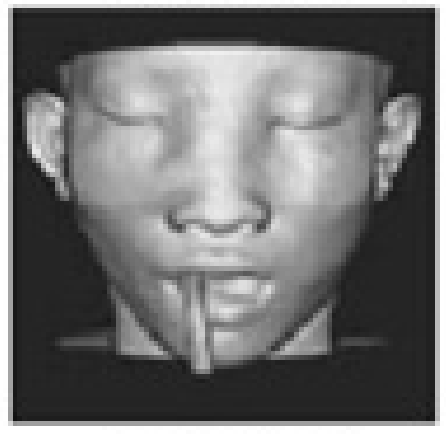

(e) $90.3 \%$

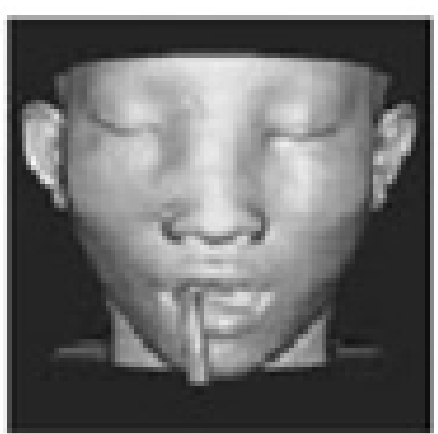

(c) $50.5 \%$

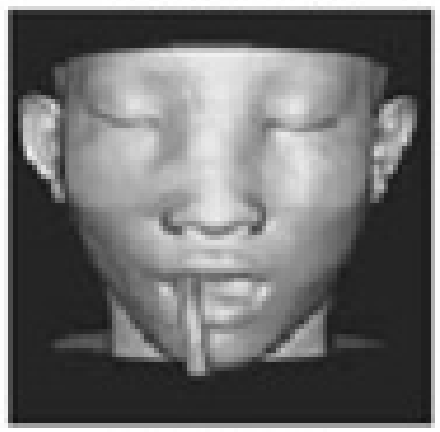

(f) $100 \%$

FiguRE 1: Progressive skin level renderings using the binary sweepings ordering: $11.8,31.2,50.5,69.9$ and 90.3 percent of the data have been transmitted. Figure (f) is a skin level rendering of the complete, original object. 
the illustration: this binary sweepings ordering can produce effective, overall progressive renderings, at least for this example and with skin level display. Progressive imaging for a local internal feature, such as a tumor within the head or multiple sclerosis lesions, requires a different analysis.

The example above illustrates fundamental features for progressive transmission of images:

1. a digital image (object) is given;

2. a decomposition of the object into subsets is made;

3. some strategy is selected to determine an ordering for transmitting the subsets; and

4. a rendering scheme for progressive imaging is chosen for subsequent use at the recipient node.

In the remainder of this section, the decomposition and strategy selection steps are determined from analysis of the FT of the object, or of its ST.

For the head CT data set (5), the discrete Fourier transform of any slice $N_{s}$ is again a matrix, $\left(A_{i j}^{(s)}\right)$, of order 128 , and the energy of the slice $N_{s}$ is

$$
E_{s}=\sum_{i=1}^{128} \sum_{j=1}^{128}\left|A_{i j}^{(s)}\right|^{2} .
$$

A straightforward scheme for progressive transmission of the $N_{s}$ may be devised using the points in transform space to calculate the energies (6): ordering can be simply that of decreasing order of the $\left\{E_{s}\right\}_{s=0}^{92}$, the first slice transmitted is one with highest energy, and so on. But, this produces poor visual results, since slices near the top of the head, being physically larger, have higher computed energies. In fact, this slice energy ordering is 0,92 , 


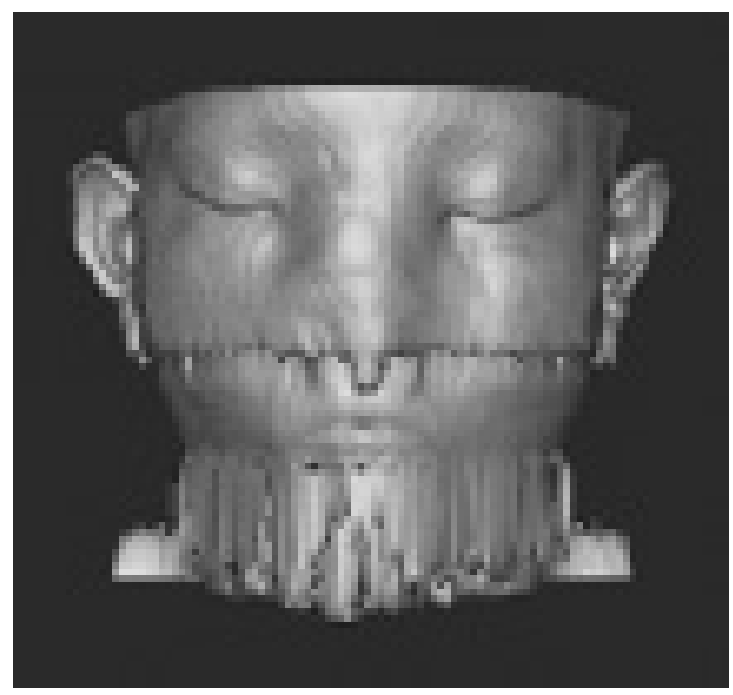

FiguRE 2: A skin rendering using the slice energies ordering scheme: the first $49.5 \%$ of the data are transmitted.

$16,15,14,17,18,19, \ldots, 83,86,87,88,89,90,91$. Transmitting the first 46 of these yields the (matrix cubic spline) skin rendering given in Figure 2.

Figure 2 shows that using the points in transform space with (6) gives a poor progressive imaging scheme; skin level rendering obscures internal detail as well. However, the individual transform elements can be employed differently to produce schemes which do focus on internal data or features. Generally, rather than summing the squares of the moduli of the points, they can be arranged in decreasing order, then the inverse transform applied to the elements corresponding to some initial segment of the sequence. This scheme may be applied to any two- or higher dimensional data set. For simplicity, both in a more detailed description of it and in illustrations, the remainder of this section focuses on a 2D data set, using an FT and an ST.

For a selected 2D discrete image $I(x, y)$, such as one of the slices $N_{s}$ in (5), its Fourier transform $F\left(k_{x}, k_{y}\right)$ can provide a progressive scheme useful for 
Algorithm 2 Transform based progressive imaging.

1: Determine the decreasing order of the moduli of the elements of $F\left(k_{x}, k_{y}\right)$,

2: Retain the first d percent of them under this ordering,

3: Insert zeros for the other elements of $F\left(k_{x}, k_{y}\right)$, then

4: Compute the inverse Fourier transform of this modified matrix.

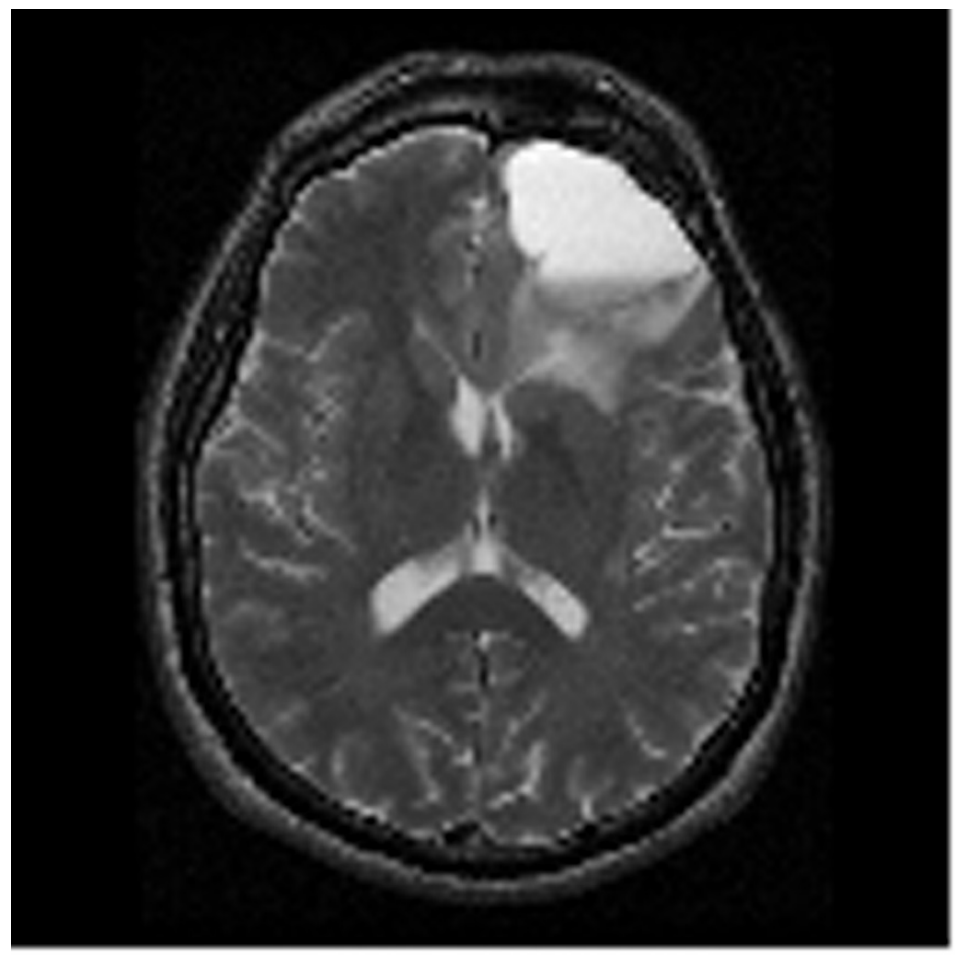

FigURE 3: MR slice of a head, with a large tumor in the upper, right-hand quadrant. Resolution $128 \times 128$. 
internal detail as follows. Consider the MR slice $I(x, y)($ a $128 \times 128$ matrix $)$ shown in Figure 3, and in the Fourier domain apply Algorithm 2.

This produces an image for rendering which incorporates precisely $d \%$ of the elements of $F\left(k_{x}, k_{y}\right)$. Results are displayed in Figure $4 \mathrm{a}-\mathrm{c}$. Note that even with just $10 \%$ of the FT data used this way, the resulting image, at least visually, reflects much of the detail of the original shown in Figure 3.

The final concept introduced in this paper entails progressive imaging based on information in a transform space, but with a capability to also include focusing on an internal feature. The $S$-transform is well suited for such localized analysis [5]. Again for simplicity, the strategy will be described around an illustration, the MR slice displayed in Figure 3, and the region of interest, the tumor. Consider, then, the $128 \times 128$ matrix $I(x, y)$ illustrated in Figure 3. Property (3) will be of use with the sT (2): the double integral (double sum in machine implementation), over the two spatial variables $x$ and $y$, of $S\left(x, y, k_{x}, k_{y}\right)$ yields the Fourier transform $\mathcal{F}\{I\}\left(k_{x}, k_{y}\right)$. Now, select in the image a region of interest (ROI) $R$, such as would be obtained by drawing any simple closed curve around the tumor slice in Figure 3. Compute $S\left(x, y, k_{x}, k_{y}\right)$, the ST of $I(x, y)$. Then, for parameter $\lambda \in[0,1]$, the matrix function

$$
\begin{aligned}
H\left(\lambda ; k_{x}, k_{y}\right)= & (1-\lambda) \iint_{I-R} S\left(x, y, k_{x}, k_{y}\right) d x d y \\
& +\lambda \iint_{R} S\left(x, y, k_{x}, k_{y}\right) d x d y,
\end{aligned}
$$

provides a family of spectra with capability for an ROI emphasis. For a selected $\lambda \in[0,1]$, Algorithm 2 (but with $F\left(k_{x}, k_{y}\right)$ replaced by $H\left(\lambda ; k_{x}, k_{y}\right)$ ) can be applied in order to give a progressive imaging scheme based on (7). Results for $\lambda=0.8$ are shown in the second row, Figure $4 \mathrm{~d}-\mathrm{f}$, and can be compared visually, and favorably, with those in Figure $4 \mathrm{a}-\mathrm{c}$. Of course, Figure $4 \mathrm{a}-\mathrm{c}$ is the result of a specific instance of $(7)$, since $2 H\left(1 / 2 ; k_{x}, k_{y}\right)=$ $F\left(k_{x}, k_{y}\right)=\mathcal{F}\{I\}\left(k_{x}, k_{y}\right)$. See the next section for comments about the choice of $\lambda$ in (7). 

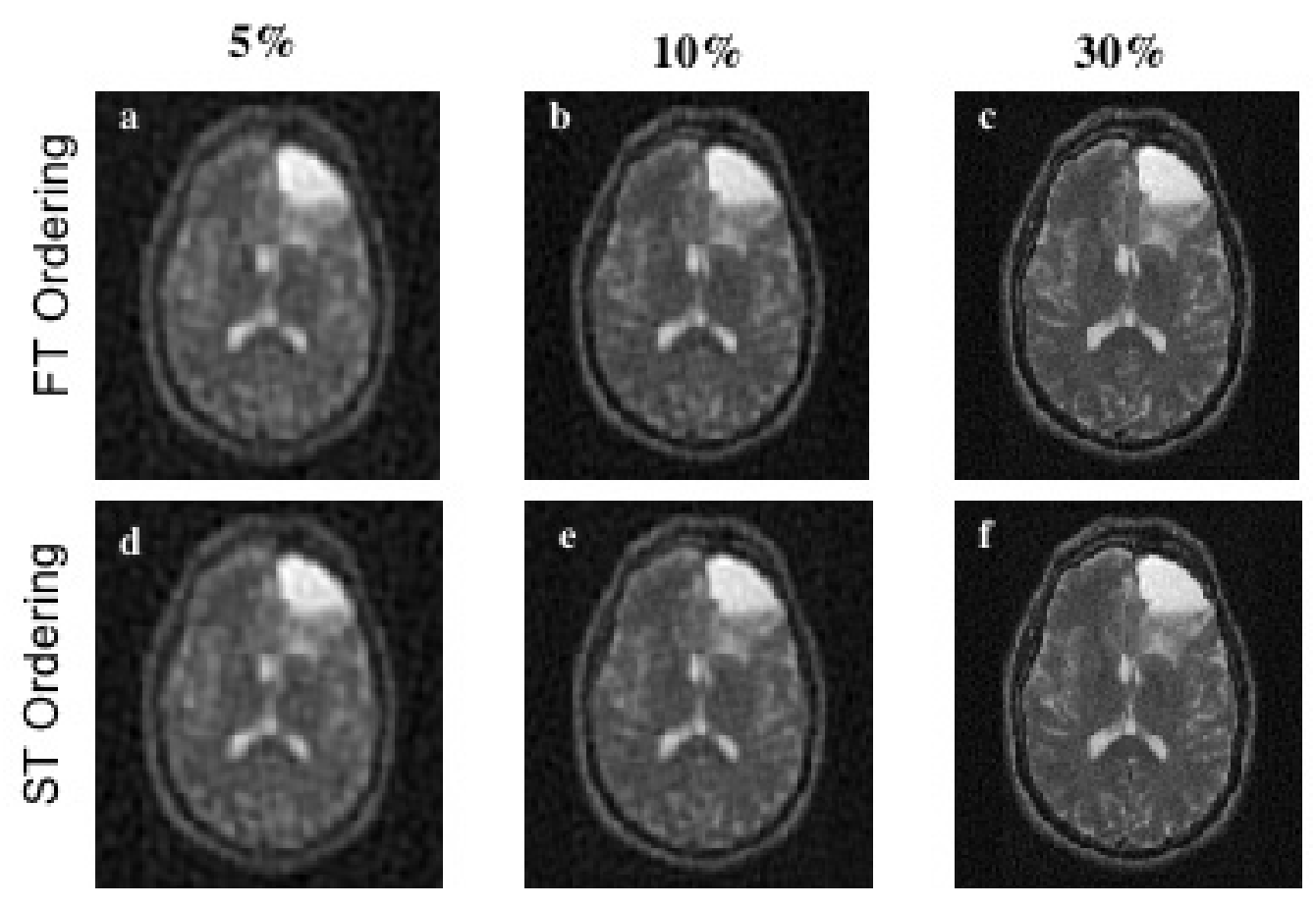

Figure 4: Progressive renderings of the original MR slice, $I(x, y)$ in Figure 3. Figure 4a-c: using "point energy" ordering in FT space, $d=5,10$ and 30 percent of the original data are employed. Figure $4 \mathrm{~d}-\mathrm{f}$ : using the ST-based mechanism (7) and Algorithm 2, d = 5, 10, and 30 percent of the elements of $H\left(\lambda ; k_{x}, k_{y}\right)$ are employed. Here $\lambda=0.8$. 


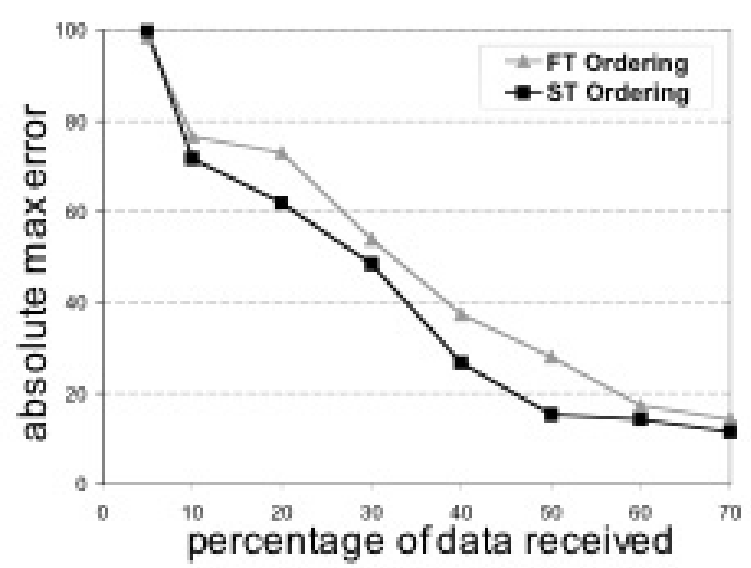

Figure 5: Maximum absolute difference between the original (Figure 3) and either (a) an FT ordering (Algorithm 2) progressive reconstruction or (b) an sT ordering (equation (7) with $\lambda=0.8$ ) progressive reconstruction. Computation restricted to the ROI, the tumor, in Figure 3 and 4.

Examination of the two rows in Figure 4, vis-a-vis the rendering in Figure 3, permits visual comparison for results of these two, transform based, progressive transmission schemes. A numerical comparison of the two schemes can also be performed, in several ways, for systematic analysis. One way simply compares norms of the differences between the full, original data set and evolving reconstruction of it - the Frobenius norm has been used recently [2]. Or, for a selected ROI, such as the tumor in Figures 3 and 4, numerical comparison can be made when a norm calculation is restricted to the subregion. This latter is the case presented in Figure 5: for this ROI, the error is smaller for the ST-based progressive scheme than for the FT one, when the amount of data transmitted lies in the $10 \%-70 \%$ range. 


\section{Concluding comments}

The paper introduces two transform based techniques for progressive transmission of images. For retention of internal detail in the data set, progression using the FT is discussed, and illustrated in Figure 4a-c. Then, for the more general situation of detail retention which also includes a capability of a region of interest emphasis, the ST is employed to obtain the basis (7) and used with the same MR data illustration (Figure 3), to produce Figure 4d-f. A numerical assessment process is illustrated in Figure 5.

The work identifies several important questions for future investigation.

1. The slice energy ordering illustrated in Figure 2 performs poorly when compared with the direct binary sweeping scheme of Figure 1, especially if it is an overall skin rendering which is used for the gauge. 3Drenderings which focus on internal local features may provide quite different results, but construction of such encompasses some unresolved problems currently.

2. In MR or CT imaging, the data are usually collected as planar slices of the physical subject. For 3D progressive imaging that is to retain internal data or features, however, decomposition of the digital image into constituent subsets which are more appropriate for the transmission scheme is still unresolved.

3. The choice of $\lambda$ in (7) is intriguing: empirical results to date indicate that $\lambda$ in the range $[0.7,0.8]$ seems to be "optimal" in the sense of the size of the maximum difference between rendering elements and those of the original image. The "Fourier" choice $\lambda=1 / 2$ appears not to be best in this sense, but an analytic solution to this optimization problem remains elusive. 
Acknowledgements: We are pleased to acknowledge the funding support from the Natural Sciences and Engineering Research Council and the Multiple Sclerosis Society of Canada, the Spanish Ministerio de Ciencia y Tecnología and FEDER grant TIC2002-02249, and the continued support of the Seaman Family Mr Research Centre in Calgary, Canada. We also acknowledge the referees for their comments and suggestions.

\section{References}

[1] R. A. Brown, H. Zhu, and J. R. Mitchell. Distributed vector processing of the $S$-transform for medical applications. Proceedings of the Annual IEEE CCECE, 2:1129-1133, 2002. C1006

[2] E. Defez, A. Law, J. Villanueva-Oller, and R. J.Villanueva. Matrix cubic splines for progressive transmission of images. J. of Math. Imaging and Vision, 17:41-53, 2003. C1004, C1007, C1014

[3] Y-S. Kim and W-Y. Kim. Reversible decorrelation method for progressive transmission of 3D medical image. IEEE Trans. Medical Imaging, 17(3):383-394, 1998. C1004

[4] W. Schroeder, K. Martin, and B. Lorensen. The Visualization Toolkit: An Object-oriented Approach to Graphics, Prentice Hall, 1997. C1006

[5] H. Zhu, B. G. Goodyear, M. L. Lauzon, R. A. Brown, G. Mayer, A. G. Law, L. Mansinha, and J. R. Mitchell. A new local multiscale Fourier analysis for MRI. Med. Phys., 30(6):1134-1141, 2003. C1004, C1005, C1006, C1012

[6] H. Zhu, X. Wei, Y. Zhang, G. S. Mayer, and J. R. Mitchell. Temporal texture analysis of normal appearing white matter in Multiple Sclerosis. Proceedings of the 11th Annual Meeting of ISMRM, Canada, p.227, 2003. C1005 